\title{
Birkhoff periodic orbits for twist maps with the graph intersection property
}

\author{
DAVID BERNSTEIN \\ Department of Mathematics, University of California, Berkeley, California 94720, USA \\ (Received 5 March 1984 and revised 16 May 1984)
}

\begin{abstract}
In this paper we show that Birkhoff periodic orbits actually exist for arbitrary monotone twist maps satisfying the graph intersection property.
\end{abstract}

\section{Introduction}

The natural condition in the theory of invariant curves for twist maps of an annulus is the so called graph intersection property (cf. [9], [10] and $\S 3$ below). This condition allows one to establish for small perturbations of integrable twist maps the existence of invariant curves with rotation numbers which are not too well approximable by rationals. As was discovered recently by Aubry, Mather [1], [8], (see also [5]) for the remaining numbers those circles are replaced by invariant order-preserving Cantor sets. As noticed in [5] those sets as well as invariant circles can be obtained as limits of order-preserving periodic orbits (Birkhoff orbits, see $\S 2$ ). This approximation process depends only on the twist condition [5], [6]. However the existence of Birkhoff periodic orbits has been established under assumptions stronger than the intersection property, namely the preservation of area [1], [8], or at least a measure positive on open sets [5]. In this paper we fill the gap and show that Birkhoff periodic orbits (and consequently the Cantor sets of Aubry-Mather type) actually exist for arbitrary monotone twist maps satisfying the graph intersection property. Incidentally the classical result of Birkhoff concerning the existence of periodic orbits with a given rotation number for area preserving maps [2] has been extended by $P$. Carter [3] to the maps satisfying an intersection property which is slightly stronger than the graph intersection property. However it was not clear whether for monotone twist maps those orbits are actually order preserving. When this work was completed the author learned about a result by G. Hall [4], obtained independently about the same time. G. Hall proved that for a monotone twist map the existence of a periodic orbit implies the existence of a Birkhoff orbit with the same rotation number. Combining the article of Hall and Carter one obtains a slightly weaker version of our theorem. It seems that our method is simpler and more straightforward than that of Hall.

\section{Notation}

We reproduce with minor modifications some background material from [5]. 
Let $A=S^{\prime} \times[0,1]=\{(\phi, r) \mid \phi \in \mathbb{R} / \mathbb{Z}, 0 \leq r \leq 1\}$ be the standard annulus, $S=$ $\mathbb{R} \times[0,1]=\{(x, y] \mid x \in \mathbb{R}, 0 \leq y \leq 1\}$ its universal covering and $T: S \rightarrow S$ the unit translation $T(x, y)=(x+1, y)$. For any homeomorphism $f: A \rightarrow A$ its lift to $S$ is defined up to a power of $T$; conversely if $F: S \rightarrow S$ and $F$ commutes with $T$ then $F$ is a lift of a homeomorphism of $A$. Let us write such an $F$ in the coordinate form: $F(x, y)=\left(F_{1}(x, y), F_{2}(x, y)\right.$. We call a homeomorphism $f: A \rightarrow A$ a monotone twist homeomorphism (or a monotone twist map) if it preserves orientation, preserves boundary components of $A$, and if for a lift $F$ of $f$ and for any $x \in \mathbb{R}$ the function $F_{1}(x, y)$ is a strictly monotone function of $y$. Obviously all functions $F_{1}(x, y)$ for different $x$ must be either increasing or decreasing. Accordingly we may speak of right or left twist maps. If $f$ is a right twist map then $f^{-1}$ is a left twist map and vice versa. In addition if $E$ denotes the involution of $A, E(x, y)=(-x, y)$, then $f$ is a right twist map if and only if $E f E$ is a left twist map. We will associate with a right twist map the following object:

The twist interval is $\left[\alpha_{0}(f), \alpha_{1}(f)\right]$, where $\alpha_{0}(f)$ and $\alpha_{1}(f)$ are rotation numbers of $F$ restricted to $\mathbb{R} \times\{0\}$ and $\mathbb{R} \times\{1\}$ correspondingly. Twist interval is defined up to an integral translation (depending on a lift $F$ ).

\section{Birkhoff periodic orbits}

Let $f: A \rightarrow A$ be a right twist map and $F: S \rightarrow S$ be a lift of $f$. If $f^{q} z=z$ for $q>0$ then there exists a unique integer $p$ such that $F^{q} w=T^{p} w$ for any lift $w$ of $z$. The fraction $p / q$ is called the rotation number of the periodic point $z$. Let $p$ and $q$ be relatively prime. We will call a point $z \in A$ a Birkhoff periodic point of type $(p, q)$ if for a lift $w$ of $z$ there exists a map $\theta=(\phi, r): \mathbb{Z} \rightarrow S$ such that

$$
\theta(0)=w, \quad \theta(n+q)=T \theta(n), \quad \theta(n+p)=F \theta(n),
$$

and $\phi$ is an increasing function. If $z$ is a Birkhoff periodic point of type $(p, q)$ then any such map $\theta$ is called a Birkhoff periodic orbit of type $(p, q)$. Obviously any Birkhoff point of type $(p, q)$ is a periodic point with rotation number $p / q$. Moreover if we denote for such a point $z, f^{n} z=\left(\phi_{n}, r_{n}\right), n=0,1, \ldots, q-1$, then all $\phi_{n}$ 's are different and lie on the circle in the same order as successive images of a point under the rotations by $2 \pi p / q$.

\section{Birkhoff theorem}

Let $f: A \rightarrow A$ be a right twist map and $F: S \rightarrow S$ be a lift of $f$. We say that $f$ has the graph intersection property if for every continuous function $h: S^{\prime} \rightarrow[0,1]$ the image $f \Gamma$ of its graph $\Gamma$ intersects $\Gamma$. It means that for every graph $\Gamma$ its image $f \Gamma$ cannot lie strictly above or below $\Gamma$. For example if $f$ is conservative, i.e. for every open set $U \subset A$ there is $n>0$ such that $f^{n} U \cap U \neq \varnothing$, then $f$ has the graph intersection property. For convenience we will always work with the universal covering instead of the annulus. In addition let us extend $F$ to a homeomorphism of the plane $\mathbb{R} \times \mathbb{R}$ by the formulae:

$$
F^{\prime}(x,-r)=\left(F_{1}(x, 0)-r,-r\right), \quad F^{\prime}(x, 1+r)=\left(F_{1}(x, 1)+r, 1+r\right), \quad r>0 .
$$


In this case $F_{2}^{\prime}(x, y)$ is a periodic function of $x$ and for any $x F_{1}^{\prime}(x, y)$ is an increasing function of $y$. Besides for any $x \lim _{|y| \rightarrow \infty}\left|F_{1}^{\prime}(x, y)\right|=\infty$. So $F^{\prime}$ is a lift of a homeomorphism $f^{\prime}$ of the cylinder $S^{\prime} \times \mathbb{R}$, every circle $S^{\prime} \times\{r\}, r \notin(0,1)$, is invariant and the rotation number $\alpha_{r}\left(f^{\prime}\right)$ of $F^{\prime}$ restricted to $\mathbb{R} \times\{r\}$ is a non-decreasing function on $\mathbb{R} \backslash(0,1)$, with $\lim _{|r| \rightarrow \infty}\left|\alpha_{r}\right|=\infty$. If $p$ and $q$ are relatively prime, $p / q \in\left(\alpha_{0}, \alpha_{1}\right)$, and $z$ is a Birkhoff point of type $(p, q)$ for $f^{\prime}$ then $z$ must belong to the annulus. So this extension does not create new Birkhoff points. Moreover if $f$ has the graph intersection property so does $f^{\prime}$. We will always assume that $F$ has been extended to $F^{\prime}$ and denote $F^{\prime}$ by the same letter $F$. For any two numbers $x, y \in \mathbb{R}$ let $I_{x}=\{x\} \times \mathbb{R}$, $I_{y}=\{y\} \times \mathbb{R}$. By the twist property the curve $F I_{x}$ meets $I_{y}$ at exactly one point $(y, s)$ and the curve $F^{-1} I y$ meets $I_{x}$ at exactly one point $(x, r)$ so $F(x, r)=(y, s)$. Denote $s$ by $H_{1}(x, y)$ and $r$ by $H_{2}(x, y)$.
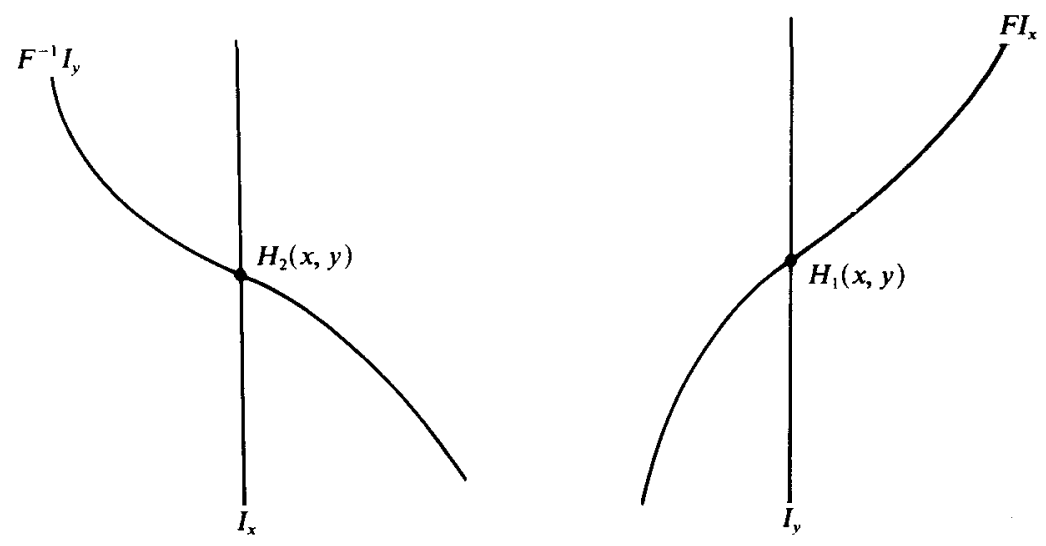

FigURE 1

Obviously $H_{1}(x, y)$ is a decreasing function of $x$ and $H_{2}(x, y)$ is an increasing function of $y$. Now consider the space $\Phi_{p, q}$ of all non-decreasing maps $\phi: \mathbb{Z} \rightarrow \mathbb{R}$ such that $\phi(n+q)=\phi(n)+1$. Let $T$ denote the unit translation: $(T \phi)(n)=\phi(n)+1$. Identifying $\phi$ with $T^{k} \phi$ for all $k$ we get the factor space $\bar{\Phi}_{p, q}$ with the natural projection $\pi: \Phi_{p, q} \rightarrow \bar{\Phi}_{p, q}$. There is a natural metric on $\Phi_{p, q}$ :

$$
d(\phi, \psi)=\max (|\phi(n)-\psi(n)|)
$$

and an induced metric on $\bar{\Phi}_{p, q}$ :

$$
\bar{d}(\bar{\phi}, \bar{\psi})=\min \left(d\left(\phi, T^{k} \psi\right)\right) .
$$

Obviously $\bar{\Phi}_{p, q}$ is compact. For every set $P \subset \Phi_{p, q}$ denote its projection into $\bar{\Phi}_{p, q}$ by $\bar{P}$. We will often use the following simple statement: if $P \subset \Phi_{p, q}$ is a $T$-invariant closed set and $m(\phi)$ is a $T$-invariant continuous function on $\Phi_{p, q}$ then $m$ reaches its minimum and maximum values on $P$.

Finally for any $\phi \in \Phi_{p, q}$ let

$$
H_{n}(\phi)=H_{2}(\phi(n), \phi(n+p))-H_{1}(\phi(n-p), \phi(n)) .
$$

Obviously $H_{n}(\phi)=H_{n+q}(\phi)$ and if $z_{n}=(\phi(n), r(n))$ is a Birkhoff periodic orbit of type $(p, q)$ then $H_{n}(\phi)=0$ for all $n$. Conversely if $\phi \in \Phi_{p, q}$ and $H_{n}(\phi)=0$ for all $n$ 
then $z_{n}=\left(\phi(n), H_{2}(\phi(n), \phi(n+p))\right.$ is a Birkhoff periodic orbit of type $(p, q)$. In this case we say that $\phi$ represents a Birkhoff periodic orbit of type $(p, q)$.

Let $\phi \in \Phi_{p, q}$. Consider $q$ numbers $H_{1}(\phi), \ldots, H_{q}(\phi)$. Let $m_{1}(\phi) \leq m_{2}(\phi) \leq \cdots \leq$ $m_{q}(\phi)$ be a non-decreasing reordering of these numbers, so $m_{1}(\phi)=\min H_{n}(\phi)$, $m_{q}(\phi)=\max H_{n}(\phi)$. Obviously $m_{i}(\phi)$ are continuous $T$-invariant functions. Additionally $m_{1}(\phi)=m_{q}(\phi)$ if and only if $H_{n}(\phi)$ has the same value for all $n$ and $m_{1}(\phi)=m_{q}(\phi)=0$ if and only if $\phi$ represents a Birkhoff periodic orbit.

LeMmA 1. Let $\phi \in \Phi_{p, q}$ and $\varepsilon>0$. If $m_{1}(\phi) \geq \alpha$ and $m_{q}(\phi)>\alpha$ then there is a point $\psi \in \Phi_{p, q}$ such that $m_{1}(\psi)>\alpha$ and for every $n, \psi(n)<\psi(n+1)$ and $\phi(n)<\psi(n)<$ $\phi(n)+\varepsilon$.

Proof. Let

$$
U=\left\{\psi \in \Phi_{p, q} \mid m_{1}(\psi) \geq \alpha, m_{q}(\psi)>\alpha, \phi(n) \leq \psi(n)<\phi(n)+\varepsilon \text { for all } n\right\} .
$$

We want to find $\psi \in U$ such that for every $n$

$$
\phi(n)<\psi(n), \quad \psi(n)<\psi(n+1), \quad H_{n}(\psi)>\alpha .
$$

Suppose $\psi \in U$ and for some $n, \psi(n)<\psi(n+1)$ and $H_{n}(\psi)>\alpha$. Choose $\gamma<$ $\min (\psi(n+1), \phi(n)+\varepsilon)-\psi(n)$ and replace $\psi(n)$ by $\psi(n)+\gamma$ (simultaneously replacing $\psi(n+i q)$ by $\psi(n+i q)+\gamma$ for all $i)$. We get another point $\psi_{1} \in \Phi_{p, q}$ such that $\phi(m) \leq \psi_{1}(m)<\phi(m)+\varepsilon$ for all $m$. Besides $\phi(n)<\psi_{1}(n), H_{n-p}\left(\psi_{1}\right)>$ $H_{n-p}(\psi) \geq \alpha, H_{n+p}\left(\psi_{1}\right)>H_{n+p}(\psi) \geq \alpha . H_{n}\left(\psi_{1}\right)$ may decrease but if $\gamma$ is small enough then $H_{n}\left(\psi_{1}\right)$ is still greater than $\alpha$. So $\psi_{1} \in U$ and is at least as good as $\psi$ for our purposes. Now for every $\psi \in U$ let

$$
\begin{aligned}
& A=\#\left\{i \in[1, q] \mid H_{i}(\psi)=\alpha\right\} \\
& B=\#\left\{i \in[1, q] \mid H_{i}(\psi)>\alpha, \psi(i)=\psi(i+1)\right\} \\
& C=\#\left\{i \in[1, q] \mid H_{i}(\psi)>\alpha, \psi(i)<\psi(i+1), \phi(i)=\psi(i)\right\} .
\end{aligned}
$$

Choose $\psi \in U$ with the minimal value of $A+B+C$. We claim that $A=B=C=0$ and therefore $\psi$ has the desired properties. Indeed if $C \neq 0$ then for some $n$ $\phi(n)=\psi(n), H_{n}(\psi)>\alpha, \psi(n)<\psi(n+1)$ and slightly shifting $\psi(n)$ to the right we decrease $C$ without increasing $A$ or $B$. So $C=0$. If $B \neq 0$ then for some $n H_{n}(\psi)>\alpha$ and $\psi(n)=\psi(n+1)$. Choose the largest $m$ such that $\psi(n)=\psi(m)$. So $\psi(m)<$ $\psi(m+1)$ and by monotonicity of $H_{1}(x, y)$ and $H_{2}(x, y), H_{m}(\psi) \geq H_{n}(\psi)>\alpha$. Then a small shift of $\psi(m)$ decreases $B$ without increasing $A$ or $C$. So $B=0$.

If $A \neq 0$ then for some $n H_{n}(\psi)=\alpha$. Among the numbers $H_{n}(\psi), H_{n+p}(\psi)$, $H_{n+2 p}(\psi), \ldots$, at least one is greater than $\alpha$ because $m_{q}(\psi)>\alpha, p$ and $q$ are relatively prime and $H_{i}(\psi)$ is periodic with period $q$. Let $H_{m}$ be the first number such that $H_{m-p}(\psi)=\alpha, H_{m}(\psi)>\alpha$. Since $B=0 \psi(m)<\psi(m+1)$ and a small shift of $\psi(m)$ increases $H_{m-p}$ therefore decreasing $A$ without increasing $B$ or $C$. So $A=B=C=0$.

THEOREM 1. There exists $\phi \in \Phi_{p, q}$ such that $m_{1}(\phi)=m_{q}(\phi)$ or, equivalently, $H_{n}(\phi)$ has the same value for all $n$.

Proof. Since $m_{1}$ is continuous and $T$-invariant on $\Phi_{p, q}$ it reaches its maximum value $\alpha$ at some point $\phi$. If $m_{q}(\phi)>\alpha$ then by lemma 1 there is a point $\psi \in \Phi_{p, q}$ such 
that $m_{1}(\psi)>\alpha$ which contradicts the assumption that $\alpha$ is the maximum value of $m_{1}$. So $m_{1}(\phi)=m_{q}(\phi)$.

Now we are ready to prove our principal result.

THEOREM 2. If $f$ is a right twist map with the graph intersection property and $p / q$ belongs to the twist interval $\left[\alpha_{0}(f), \alpha_{1}(f)\right]$, where $p$ and $q$ are relatively prime, then $f$ has a Birkhoff periodic point of type $(p, q)$.

Proof. As was mentioned before, we can assume that $f$ has been extended to a twist map of the cylinder. By theorem 1 there is a point $\phi_{0} \in \Phi_{p, q}$ such that $m_{1}\left(\phi_{0}\right)=$ $m_{q}\left(\phi_{0}\right)=\alpha$. If $\alpha=0$ then $\phi_{0}$ represents a Birkhoff periodic orbit. Suppose $\alpha \neq 0$. It suffices to consider the case when $\alpha>0$. Indeed if $\alpha<0$ then we consider the involution $D: \mathbb{R} \times \mathbb{R} \rightarrow \mathbb{R} \times \mathbb{R}, D(x, r)=(-x, 1-r)$ and replace $F$ by the map $F^{\prime}=$ DFD. $F^{\prime}$ is a right twist map with the graph intersection property and it suffices to prove the existence of a Birkhoff periodic orbit for $F^{\prime}$. On the other hand $\psi_{0}(n)=$ $\left(-\phi_{0}(-n)\right)$ has the property that $m_{1}\left(\psi_{0}\right)=m_{q}\left(\psi_{0}\right)=-\alpha>0$. So we can assume that $m_{1}\left(\phi_{0}\right)=m_{q}\left(\phi_{0}\right)=\alpha>0$. Now consider the set

$$
P=\left\{\phi \in \Phi_{p, q} \mid m_{1}(\phi) \geq 0\right\} .
$$

$P$ is a closed $T$-invariant subset of $\Phi_{p, q} . P$ is non-empty since it contains $\phi_{0} . m_{q}$ is continuous and $T$-invariant so it reaches its minimum value $\beta$ on $P$ at some point $\phi_{1} \in \Phi_{p, q}: m_{q}\left(\phi_{1}\right)=\beta \geq m_{1}\left(\phi_{1}\right) \geq 0$. If $\beta=0$ then $m_{1}\left(\phi_{1}\right)=m_{q}\left(\phi_{1}\right)=0$ and $\phi_{1}$ represents a Birkhoff periodic orbit.

To complete the proof it suffices to assume that $\beta>0$ and to construct a graph $\Gamma$ whose image $F \Gamma$ lies strictly below $\Gamma$ which will contradict the assumption that $f$ has the graph intersection property. To construct such a graph we will produce a continuous family of points $\phi_{t} \in P$ such that for every $n \phi_{t}(n)$ is an increasing function of $t$. This family will also have some periodicity property, namely for some positive integers $k, N$

$$
\phi_{t+k}(n)=\phi_{t}(n)+N \quad \text { for all } n, t .
$$

First consider the following two sets:

$$
P_{1}=\left\{\phi \in \Phi_{p, q} \mid m_{1}(\phi) \geq \beta / 2\right\}, \quad P_{2}=\left\{\phi \in \Phi_{p, q} \mid m_{1}(\phi) \geq \beta / 4\right\} .
$$

Note that $\phi_{0} \in P_{1} \subset P_{2}$. Both $P_{1}$ and $P_{2}$ are closed, $T$-Invariant subsets of $\Phi_{p, q}$. Since $m_{1}$ is continuous and $\bar{P}_{1}, \bar{P}_{2}$ are compact there is $\varepsilon>0$ such that if $\phi \in P_{1}$ and $d(\phi, \psi)<2 \varepsilon$ then $\psi \in P_{2}$. In particular for any two points $\phi, \psi \in P_{1}$ with $d(\phi, \psi)<2 \varepsilon$ their linear interpolation

$$
\phi_{t}(n)=t \phi(n)+(1-t) \psi(n) \quad(0 \leq t \leq 1)
$$

represents a curve in $P_{2}$.

Lemma 2. There exists $\delta>0$ such that for every $\phi \in P_{1}$ there exists $\psi \in P_{1}$ such that for all $n$

$$
\phi(n)+2 \delta<\psi(n)<\phi(n)+\varepsilon .
$$

Proof. Let $\phi \in P_{1}$ then $m_{1}(\phi) \geq \beta / 2$. On the other hand $m_{q}(\phi) \geq \beta>\beta / 2$ so by lemma 1 there is $\psi \in P_{1}$ such that for all $n \phi(n)<\psi(n)<\phi(n)+\varepsilon$. Let 
$\delta(\phi)=\frac{1}{4} \min (\psi(n)-\phi(n))$. For all $n$

$$
\phi(n)+2 \delta(\phi)<\psi(n)<\phi(n)+\varepsilon
$$

Moreover there exists a neighbourhood $U(\phi)$ such that for every $\phi^{\prime} \in U$ and for all $n \phi^{\prime}(n)+2 \delta(\phi)<\psi(n)<\phi^{\prime}(n)+\varepsilon$. Since $\vec{P}_{1}$ is compact and $\{\bar{U}(\phi)\}$ form an open covering of $\bar{P}_{1}$ there is a finite subcovering $\left\{\bar{U}\left(\phi_{i}\right)\right\}$ of $\bar{P}_{1}$. Now set $\delta=\min \delta\left(\phi_{i}\right)$.

Next we construct a sequence of points $\phi_{i} \in P_{1}$. Start with $\phi_{0} \in P_{1}$. Using lemma 2 repeatedly construct a sequence $\phi_{i} \in P_{1}$ such that for all $n^{\prime}$

$$
\phi_{i}(n)+2 \delta<\phi_{i+1}(n)<\phi_{i}(n)+\varepsilon \text {. }
$$

Since $\bar{P}_{1}$ is compact the sequence $\bar{\phi}_{i}$ has an accumulation point, i.e. for some $m, k>0 \quad \bar{d}\left(\bar{\phi}_{m}, \bar{\phi}_{m+k}\right)<\delta$. It means that for some positive integer $N$, $\left|\phi_{m}(n)+N-\phi_{m+k}(n)\right|<\delta$ and therefore

$$
\phi_{m+k-1}(n)+\delta<\phi_{m}(n)+N<\phi_{m+k-1}(n)+2 \varepsilon .
$$

Next we extend the segment of the sequence $\phi_{m}, \phi_{m+1}, \ldots, \phi_{m+k-1}$ to the infinite sequence $\left\{\phi_{i}\right\}, i \in \mathbb{Z}$ by the formula

$$
\phi_{i+k}(n)=\phi_{i}(n)+N
$$

This sequence has the following property: $\phi_{i}(n)+\delta<\phi_{i+1}(n)<\phi_{i}(n)+2 \varepsilon$. Using linear interpolation we extend this sequence to a continuous family $\phi_{t}$ by the formula:

$$
\phi_{i+s}(n)=(1-s) \phi_{i}(n)+s \phi_{i+1}(n) \quad 0 \leq s \leq 1 .
$$

This family has the following properties:

for every $t, \phi_{t} \in P_{2}$;

for every $n, \phi_{t}(n)$ is an increasing function of $t$;

$\phi_{t+k}(n)=\phi_{t}(n)+N$.

Now we are ready to construct a graph $\Gamma$ whose image $F \Gamma$ lies strictly below $\Gamma$. Namely, for $x \in \mathbb{R}$ let

$$
h(x)=\max _{t, n}\left\{H_{2}\left(\phi_{t}(n), \phi_{t}(n+p)\right) \mid \phi_{t}(n)=x\right\} .
$$

Although there are infinitely many pairs $(t, n)$ for which $\phi_{t}(n)=x$ it suffices to consider only finitely many of them - because of periodicity properties:

$$
\phi_{t}(n+q)=\phi_{t}(n)+1 \text { and } \phi_{t+k}(n)=\phi_{t}(n)+N \text {. }
$$

For the same reason $h(x)$ is periodic: $h(x+1)=h(x)$. Since, for every $n, \phi_{t}(n)$ is an increasing function of $t, h(x)$ must be continuous. Let $\Gamma$ be the graph of $h$. If $(x, r) \in \Gamma$, i.e.

$$
r=H_{2}\left(\phi_{t}(n), \phi_{t}(n+p)\right)
$$

for some $n, t$ such that $\phi_{t}(n)=x$, and $F(x, r)=(y, s)$, then $y=\phi_{t}(n+p)$ and

$$
s=H_{1}\left(\phi_{t}(n), \phi_{t}(n+p)\right)=H_{2}\left(\phi_{t}(n+p), \phi_{t}(n+2 p)\right)-H_{n+p}\left(\phi_{t}\right) \leq h(y)-(\beta / 4) .
$$

So $(y, s)$ lies strictly below $\Gamma$ and $F \Gamma \cap \Gamma=\varnothing$.

In conclusion, let us mention an interesting generalization of theorem 2 . In the measure preserving case there are always at least two different Birkhoff orbits of 
any given type [7]. Moreover, they are compatible, i.e. for some points $\phi, \psi \in \Phi_{p, q}$ representing these orbits $\phi(n)<\psi(n)<\phi(n+1)$ for all $n$. In [7] only the minimal energy orbit was shown to have an accompanying compatible (mini-max) orbit. In general the graph intersection property does not imply the existence of two orbits. Namely, it is possible to construct a twist map $f$ with the graph intersection property that has exactly one Birkhoff orbit of type $(p, q)$. In this case, however, for some graph $\Gamma$ the image $f \Gamma$ of $\Gamma$ lies below $\Gamma$ except for finitely many points (and these points form the only Birkhoff orbit). To avoid such maps we will use the following definition. We say that $f$ has the strong graph intersection property if for every graph $\Gamma$ of a continuous function its image $f \Gamma$ either coincides with $\Gamma$ or contains points both above and below $\Gamma$. For example, every conservative map has the strong graph intersection property. Under this condition it can be shown that every Birkhoff orbit is accompanied by a compatible orbit. For an arbitrary twist map $f$ it can be shown that if there is a Birkhoff periodic orbit of type $(p, q)$ which is not accompanied by a compatible orbit then there are no other periodic orbits, Birkhoff or otherwise, of type $(p, q)$. These results will be proved in our next paper.

\section{REFERENCES}

[1] S. Aubry. Theory of the Devil's staircase. In Seminar on the Riemann Problem and Complete Integrability, 1978-79. (Ed. D. G. Chudnovsky), Lecture Notes in Math. 925, Springer: BerlinHeidelberg-New York.

[2] G. D. Birkhoff. Proof of Poincare's geometric theorem. George David Birkhoff: Collected Mathematical Papers, vol. 1 Dover Publishing Inc: New York, 1968, p. 673.

[3] P. Carter. An improvement of the Poincaré-Birkhoff fixed point theorem. Trans. Amer. Math. Soc. 269 no. 1, (1982), 285-299.

[4] G. R. Hall. A topological version of a theorem of Mather on twist maps. Ergod. Th. \& Dynam. Sys. 4 (1984), 585-603.

[5] A. Katok. Some remarks on Birkhoff and Mather twist map theorems. Ergod. Th. \& Dynam. Sys. 2 (1982), 185-194.

[6] A. Katok. Periodic and quasi-periodic orbits for twist maps. In Dynamical Systems and Chaos, Springer Lecture Notes in Physics 179 (1983) 47-65.

[7] A. Katok. More about Birkhoff periodic orbits and Mather sets for twist maps. Preprint.

[8] J. N. Mather. Existence of quasi-periodic orbits for twist homeomorphisms. Topology (1982).

[9] J. Moser. Stable and Random Motions in Dynamical Systems. Princeton Univ. Press: Princeton, 1973.

[10] H. Rüssmann. On the existence of invariant curves of twist mappings of an annulus. Preprint, 1982. 